\title{
A Bipedal Mechanical Walker with Balancing Mechanism
}

\author{
Miša STOJIĆEVIĆ, Miodrag STOIMENOV, Zorana JELI
}

\begin{abstract}
Walkers are mechanical leg devices which perform motion similar to a physiological walk of animals or humans. They have many different usages among which the application for medical rehabilitation of injured persons not capable of walking is the most important. This paper presents the mechanism embedded in a mechanical walker by which the balancing of a human mass centre is accomplished. The benefit of such kind of mechanism is that a walker device with the balanced mass centre can be supplied with the feet of a smaller size. Moreover, it generates more pleasant walking movement, similar to the physiological bipedal motion. This mechanism has been calculated, 3D modelled and its operation simulated, analysed and numerically and graphically described. Finally, the motion of the chosen referent point on the mechanical walker obtained by the simulation is compared with the motion of the corresponding referent point on the human body acquired by camera. The results of this comparison disclosed that trajectories of the chosen referent points on the mechanical walker and human body are almost overlapped. Thus, it has been proven that the mechanism proposed in this paper is capable to balance the mass centre of a human body correctly.
\end{abstract}

Keywords: balance; feet; mechanism; rehabilitation; 3d model; walker

\section{INTRODUCTION}

Walkers are mechanical leg devices which perform motion similar to a physiological walk of animals or humans. The objective of this study is the synthesis of walking mechanism by which the walking machinery can be designed and created, capable for moving on two or more legs. In particular, this paper presents the mechanism embedded in a mechanical walker by which the balancing of a human mass centre is accomplished.

The problem of mechanical walkers, their kinematical characteristics, control, synthesis and design, and different applications has been considered, discussed and presented in many publications. Thus, H. Hirukawa et al. in [1] present a motion pattern generator of humanoid robots that walk on a flat plane, tilted steps and a rough terrain. They generate the walking pattern as a solution of the contact wrench equations and by applying the revealed contact wrench condition between a humanoid robot and terrain. O. Khatib et al. in [2] establish the relationship between basic characteristics of human postural motion and potential energies of the muscles physiological activities. In [3], N. Mansard et al. apply a general framework for building complex whole-body control for highly redundant robot. They propose the implementation of the exposed control system for visually-guided grasping of a humanoid walking robot. In [4], L. Khatib et al. synthesize whole-body behaviours by controlling multiple behavioural primitives which describe and regulate various movement criteria (behaviour of the centre of gravity, hands, legs, and head, the body attitude and posture, joint-limits and contacts, etc.)

In particular, they present multi-level hierarchical control structure that establishes general priorities among behavioural primitives, and describe compliant control strategies for efficient control under contact interactions. In [5], K. Monkovaet al. expose the kinematic analysis of quick-return mechanism that is executed by three various methods. The article describes the basic principles of three approaches, as well as the advantages and disadvantages of presented solutions. In [6], Z. Ivandic et al. present a new systematic approach to evaluate kinematic structures of the robot for welding in the application of certain conceptual solutions in different welded forms of product design. They analyse five groups of welded structures with three characteristic examples in each group of products designed at the conceptual level. S. Collins et al. reveal in [7] that they have built the first three-dimensional, kneed, two-legged, passive-dynamic walking machine. Besides features of two-dimensional models, the proposed three-dimensional device has some novel constructive and functional characteristic such as specially curved feet, a compliant heel, and mechanically constrained arms to achieve a harmonious and stable gait. N. Koceska et al. consider and explain in [8] the application of pneumatically actuated robot systems in gait training.

They accentuate the high importance and benefit of this application in rehabilitation of people with locomotion disabilities such as paraplegia, quadriplegia, multiple sclerosis, muscular dystrophy, spinal cord injury, persons affected by stroke, Parkinson disease, etc. R. Andrejević et al. disclose in [9] the synthesis and fundamental kinematic analysis of mechanically established walking and standing mechanism, as well as the design and motion simulation of a mechanical walker 3D model. Authors emphasize that practical application of this mechanical walker covers the fields of walking rehabilitation and training in the treatment of spinal cord injured patients, as well as for standing and walking assistance of elderly and disabled people. In [10], M. Vukobratović et al. expose a short survey of the history of the origin of ZMP (Zero-Moment Point) and give a very detailed elaboration of the ZMP concept, with a special review concerning some characteristic boundary cases. Finally, they consider some unresolved or insufficiently treated phenomena that may yield a significant improvement in robot performance.

In [11], M. Stojićević et al. present and describe a mechanism with an approximately straight-line movement of its specific point and approximately translational movement of the mechanism component which is a part of dyad and contains the same specific and aforementioned point. They disclose the procedure for determining optimal parameters of the complete 
mechanism, including dyad, by using some of the commercial software packages (MATLAB, Solid Works).
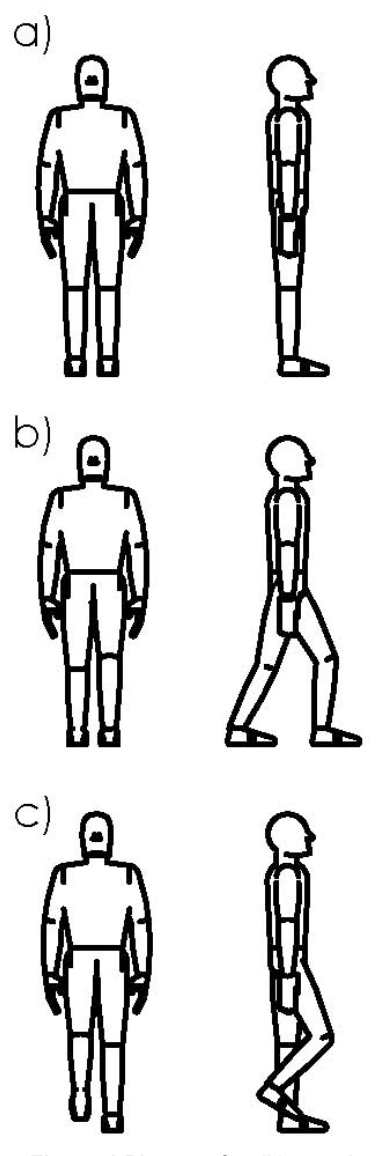

Figure 1 Phases of walking cycle

Walking machines are important and relevant, especially as a means of solving the problem of movement on the road (or area with difficult terrain). The wheel only partially solves the problem of movement, as for its efficiency use smooth surface needs to be continuous, but leg device is able to contact feet to the surface with irregularities and make a smooth transit over it. If you have a smooth, firm road, wheel surface certainly serves as a basis for most efficient vehicles. When it comes to natural surface, which comprises irregularities in many aspects wheel is useless and there may be applicable stepping device.

In Fig. 1 three phases of walking cycle can be seen: Fig. 1a shows a standing position, where body is in balance and completely stable. The next position (Fig. 1b) shows another stable position in which legs are in standing position and getting ready to move the body forward. Except these two positions, all other phases of walking are unstable (Fig. 1c). While standing on one foot, the centre of mass shifts to a side of standing foot thus creating a balance, so that swinging cycle is over and body is back in a stable position.

Based on these, we have two types of stabilities: static (when person is standing or during a step when both feet are on the ground) and dynamic (when one foot is on the ground and the other is lifted).

There are several approaches to this problem. H. Hirukawa and authors are proposing a whole body motion while body is moving [1]. Another principle is based on using external forces to act on the robot to achieve a stable walk [2], [3], [4]. Here, a mechanism for the whole body mass distribution will be described in order to achieve a stable walking.

Computer simulation plays a significant role in modelling and simulating devices that will help scientists and engineers to gain an insight into performance of their work and allow the construction of devices that will improve mobility and optimize human performance.

By using CAD software that has the ability to analyse a processing of simulation, not only a motion and trajectories can be obtained, but many other variables relevant to specific tasks. In [5] a group of authors is presenting a kinematic analysis of quick-return mechanism. Similarly, [6] have done the analysis and obtained the results for geometric synthesis for joints on their crane drive mechanism.

Modelling the human body as a series of interconnected rigid links is a standard biomechanical approach [7]. In order to make a biped robot walking anthropomorphic like, most of the leg mechanisms are built with three or more actuating motors at least with the hip, knee and ankle. These kinds of legs have anthropomorphic design and the same degrees of freedom of human legs, which give them the ability to be more flexible. A major setback for this kind of humanoid walkers is their price, which is a result of many motors and electronics implemented in them. In order to make more affordable device that will help people, authors have chosen a different approach, and as a consequence it will have a limited range of possible operations.

This is very important for persons who have locomotor dysfunctions in the lower extremities. For a paraplegic person a bipedal walker represents an overall improvement in the domain of quality of life such as physically, emotionally and socially [8]. Thus, it is important to emphasize that, among many different practical applications of a mechanical walker, one of the most important is the medical rehabilitation of injured persons not capable of walking.

Here, it will be shown how two 1 degree-of-freedom (DOF) mechanisms are used to drive a bipedal walker and to swing patient's body from one side to another and thus to achieve that a trajectory of the patient in a mechanical walker is compared with trajectories of normal walk.

\section{WALKING MECHANISM}

In order to obtain a stable position of a body in motion, it is generally necessary for a body to have a 3point support. Consequently, for the stable walking machines to be sustainable, at least three legs are needed. The creation of mobile robots that can walk on two legs as stable as humans do is extremely difficult and most of the methods are being developed for providing dynamic stability of a bipedal walking machine. The problem of dynamic stability can be observed by the motion of the two-legged toy. Usually, these toys are designed in such a way that the movement of one leg does not affect the state of the other, and the projection of the centre of gravity (COM) is always onto the area occupied by the foot of one of the legs (Fig. 2). 


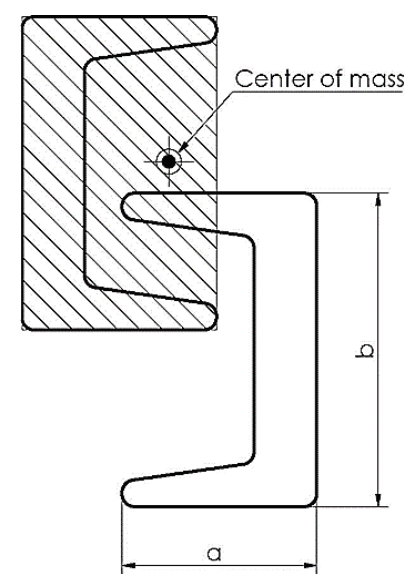

Figure 2 Feet of a mechanical walker

This solution has been used by the authors [9] and a 1:5 scale model was built (Fig. 3).

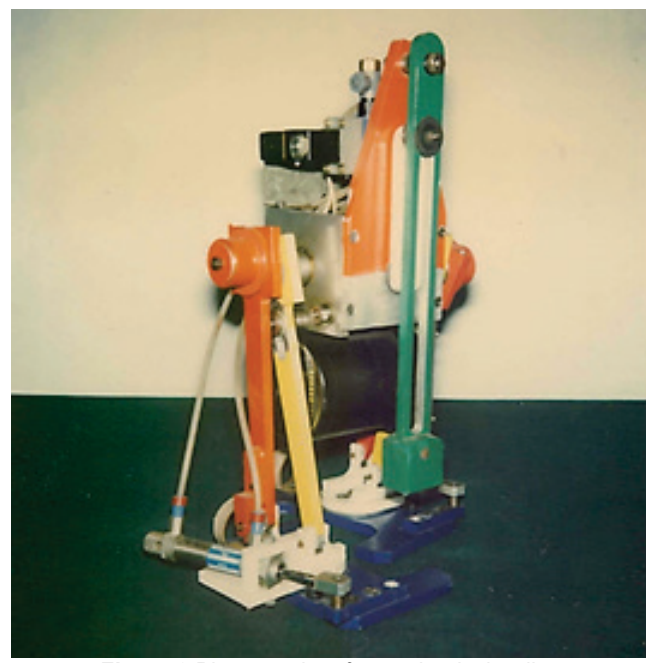

Figure 3 Photography of a mechanical walker

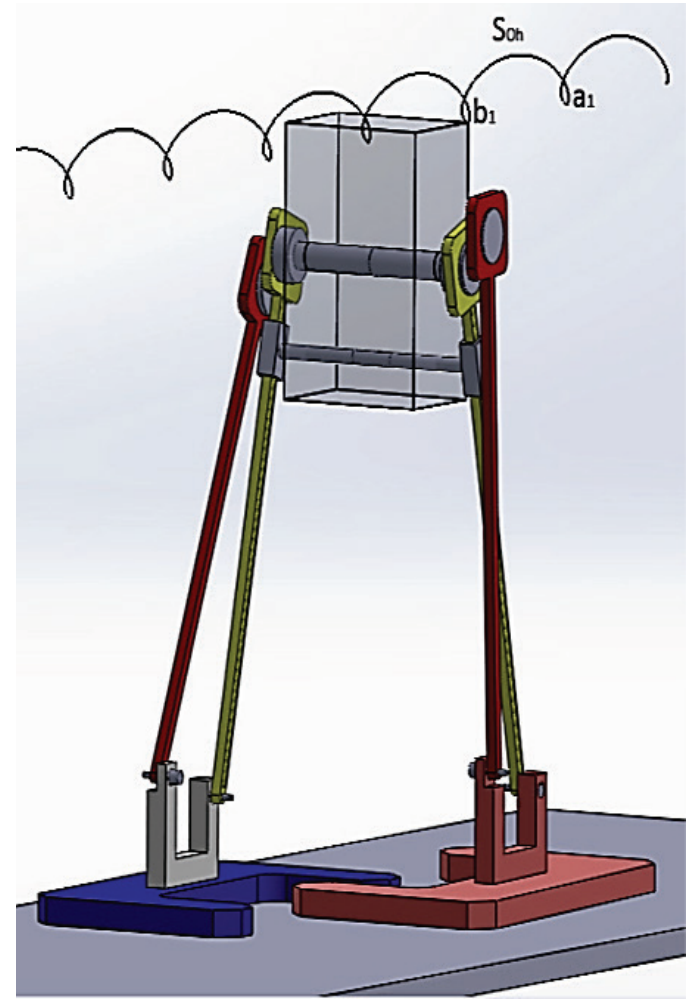

Figure 4 3D model of a mechanical walker with trajectory
This model has been transformed into the 1:1 scale using program Solidworks (Fig. 4) for further experimentation purposes. Zero moment point (ZMP) [10] is satisfied using feet shown in Fig. 2, but it lacks dynamic stability for greater speeds. There are several solutions for the balancing problem and one of those solutions is shown in Fig. 5. The movable mass $m_{2}$ is fastened to the end of a coulisse follower. One of the disadvantages attributed to this solution, which is evident, is that during motion the mass $m_{2}$ remains in the farthermost position from the zero position for a very short while.

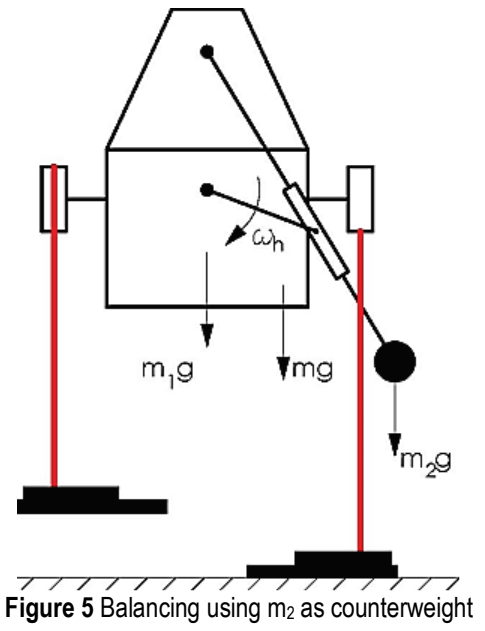

\section{BALANCING MECHANISM}

Although this solution has provided a solid basis for a scaled model, there are several better solutions for a walker and its purpose. If such walker were used as a rehabilitation aid for paralytics, a proper solution would be a mechanism that could shift the patient's body according to the law of motion, required in this case, which would provide satisfactory balancing of a complete man/walker system. One such mechanism [11] is shown in Fig. 6

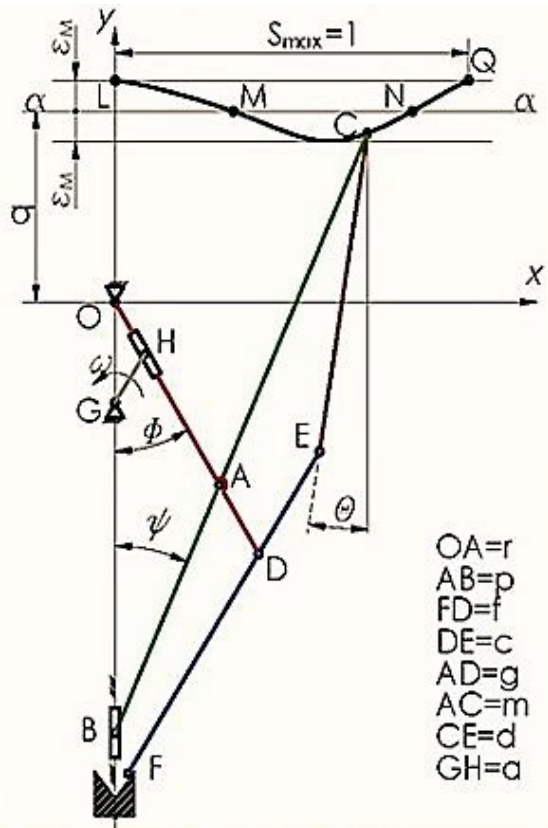

Figure 6 Mechanism with approximately straight-line path of a dyad 
Here, a mechanism is shown with an approximately straight-line movement of the point $\mathrm{C}$ and approximately translational movement of the member $\overline{C E}$ which is a part of the dyad. Member $\overline{C E}$ is used to shift patient's position and thus to slide a body weight from one side to another. Since mechanism movement is symmetrical with axes $\mathrm{O} y$, it is given $S_{\max }$, which is one a half of the required path.

Mechanism optimum parameters are those which allow the trajectory of a driven point $\mathrm{C}$ to have the same maximum deviation $\varepsilon_{m}$ above and below desired linear path $\overline{\alpha \alpha}$.

The path of a driven point $\mathrm{C}$ in the coordinate system (whose centre is at the point $\mathrm{O}$ and axis $O_{x}$ is parallel to the ideal trajectory $\overline{\alpha \alpha}$ ) is described by equations:

$y_{c}=-r \cos \phi+m \cos \psi$

$x_{c}=(p+m) \sin \psi$

The consequence of condition $p=r$ is that $\Varangle \phi=\Varangle \psi$ so that Eqs. (1) and (2) are reduced to:

$y_{c}=(m-r) \cos \psi=(m-r) \cos \phi$

$x_{c}=(p+m) \sin \phi$

Path $S_{c}$, of a driven point $\mathrm{C}$, can be affected by the changes of the parameters $r$ and $p$ in the relation, which causes a change of angles $\phi$ and $\psi$. Because of the symmetry of movement, trajectories of slider mechanisms must be maintained on the axis $O_{y}$. The change of the relations' parameters $r$ and $p$ can be twofold as follows:

a) $r>p \Rightarrow \Varangle \phi>\Varangle \psi$

b) $p>r \Rightarrow \varangle \psi>\Varangle \phi$

Parameters $\mathrm{r}$ and $\mathrm{p}$ and angles $\Varangle \phi$ and $\Varangle \psi$ are linked by the relation, which is a result of the law of sines applied to triangle OAB (Fig. 6):

$\frac{r}{\sin \psi}=\frac{p}{\sin \phi}$

For the case when $p>r$, the driven point $\mathrm{C}$ will have a path $S_{c}$ with three extreme points.

To determine the exact points (and based on these parameters of slider mechanism), we start from the following assumptions:

- $S_{\max }=1$

- $\quad$ known length $\overline{B C}=l$

- $\quad$ known distance q of line $\alpha \alpha$ from axis $O_{x}$.

The line $\alpha \alpha$ can be set in such a way that the difference between the extremal points of ordinates $2 \varepsilon_{m}$ is equally divided.

According to the Chebyshev terms [12], [13], to achieve the most accurate approximation, it is necessary that the selected value of $\varepsilon_{m}$ of curve $S_{c}$, at a distance $S_{\max }$ from the axis $O_{y}$ is also deviating from the line $\overline{\alpha \alpha}$ by the size of $\varepsilon_{m}$

Finding optimal parameters of the mechanism that meet the requirements of the most precise approximation is related to determination of the exact points $\mathrm{M}$ and $\mathrm{N}$ with the points L and Q (Fig. 6), which are on the same ordinate.

With known parameters $\mathrm{p}$ and $\mathrm{r}$, parameter $\varepsilon_{m}$ can be determined in a simple way:

$\varepsilon_{m}=l-p-r-q$

Approximation of the exact translation can be achieved if the point E (Fig. 6) on the additional dyad has a path $S_{e}$ which is exact to the trajectory path $S_{C}$.

The size of deviation $\varepsilon_{m}$ between path $S_{C}$ and line $\alpha \alpha$ (about $10^{-3} q$ ) provides the possibility to achieve an approximately straight-line translational movement of the member $\overline{C E}$.

To do this, it is first observed the mechanism that consists of two sliding mechanisms, OABC and ODFE. These mechanisms have a common rotation point $\mathrm{O}$, and points $\mathrm{O}, \mathrm{A}$ and $\mathrm{D}$ on the same member.

The mechanism could realize a precise translational movement of the member $\overline{C E}$, whose length is changing, but in that case the parameters of mechanism ODFE have to be in exact relation with the parameters of the basic mechanism OABC.

Change in length of the member $\overline{C E}$ is less than the size of $\varepsilon_{m}$ of the basic mechanism, because paths $S_{C}$ and $S_{E}$ are similar. Due to this similarity, it can be concluded that member $\overline{C E}$ can have a fixed length, but then point $\mathrm{F}$ has to be set free, or the mechanism will be a fixed figure. These mechanisms will have approximately translational movement of an additional dyad $\overline{C E}$.

For all mechanism positions (Fig. 7), e.g. for every $\phi$, with known parameters of the mechanism and additional dyad angle, deviation $\theta$ can be found.

This way of determining the mechanism parameters enables future constructors to quickly and simply determine mechanism optimum parameters with additional dyad, whose member is accomplishing approximate translation according to given demands.

\section{SIMULATION AND RESULTS}

In the last two chapters two mechanisms elaborated in [11] and [9] have been presented. By combining these two mechanisms we have a mechanical walker that has the ability to move a patient forward and backward with exact trajectory of COM, as achieved from testing normal walk. This mechanism is shown in Fig. 7, and Fig. 8 shows a patient attached to the mechanical walker. As discussed before, points $\mathrm{C}$ and $\mathrm{E}$ (shown in Fig. 6) are the points connected on two joints that keep patient in a vertical standing. Point $\mathrm{E}$ is located around the hip and point $\mathrm{C}$ is just below the armpits. Walker's feet shown in Fig. 2 and Fig. 4 are now reduced to a smaller size (dimension $a$ in Fig. 2 is significantly smaller) due to the lack of necessity to keep balance with them.

Reduced feet size, ski-like now, is more applicable in a real treatment of the patient. Both of these mechanisms are connected on the same motion shaft and thus both can have equal angular velocities providing a uniform translation. 


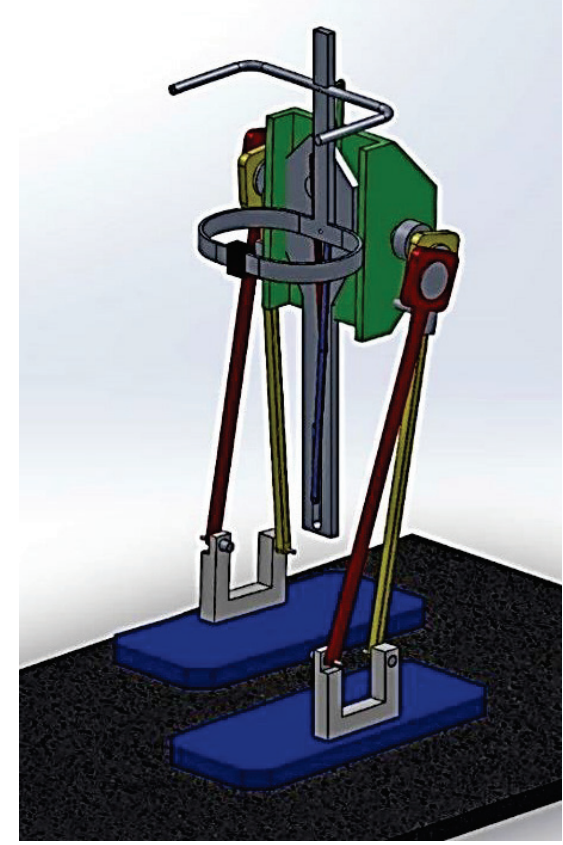

Figure 7 Mechanical walker with balancing mechanism

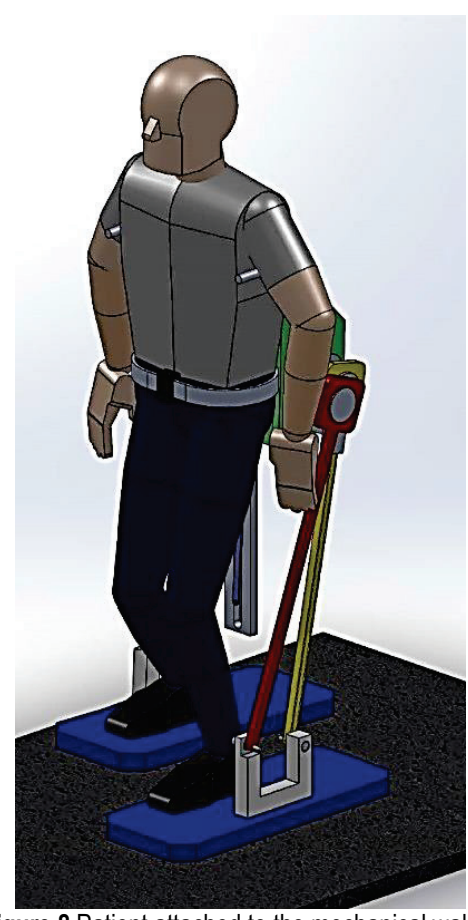

Figure 8 Patient attached to the mechanical walker

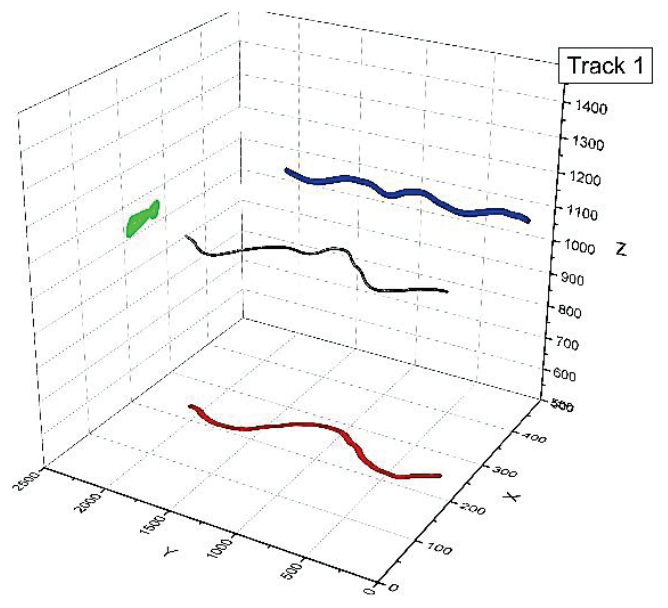

Figure 9 Track 1

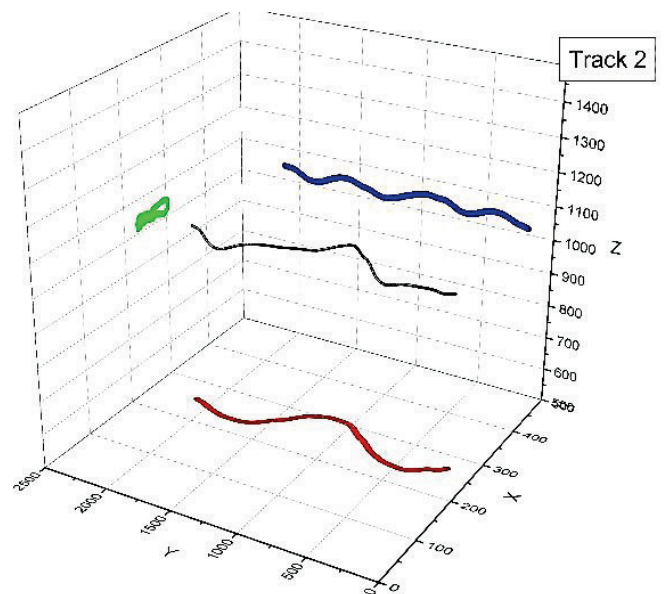

Figure 10Track 2

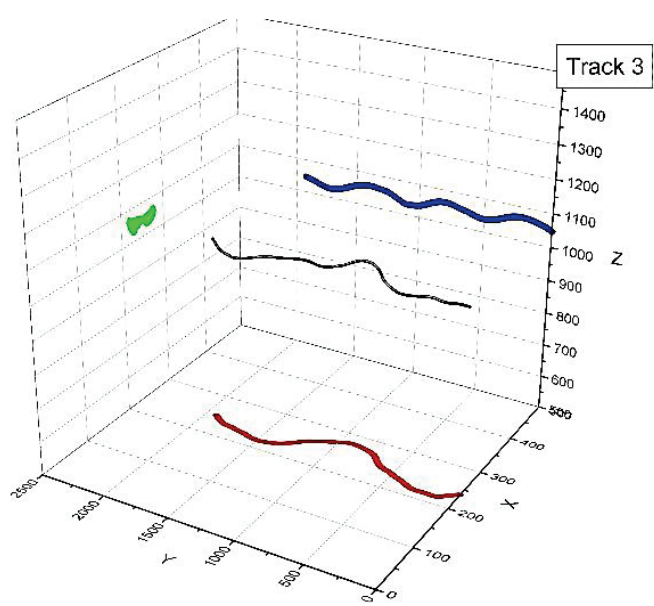

Figure 11 Track 3

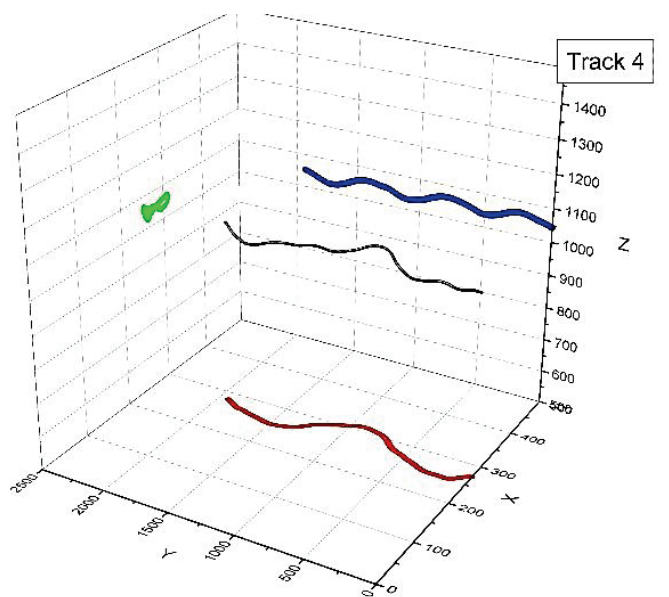

Figure 12 Track 4

Input parameters of normal human walking are obtained from healthy persons who walked at normal speed by the Vicon Motion Capture system installed at the Laboratory of Mechatronics, Robotics and Automation, at the Faculty of Technical Sciences, Novi Sad. This system is infrared marker-tracking equipment that offers millimetre resolution of $3 \mathrm{D}$ spatial displacements of human body. Its technical characteristics are displayed in Tab. 1.

A movement of the centre of mass is obtained from four different measurements on the same subject (Fig. $9 \div 12$ ). 
The centre of mass of the entire body is located slightly above the mid-height $(55 \%)$ of the individual. In a simplified analysis, the COM location is where the entire body will be balanced by Earth's gravity. In this case, for a body standing on both feet, the centre of mass is located in the middle of the hips.

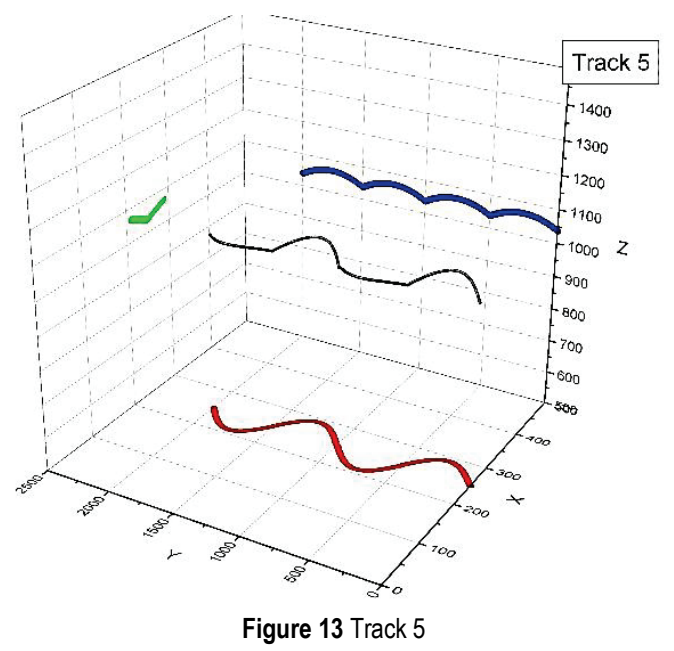

Table 1 Characteristic of Vicon Motion System

\begin{tabular}{|l|c|}
\hline Resolution (Megapixels) & 8 \\
\hline Maximum Frame Rate (fps) at full resolution & 260 \\
\hline Standard Lens & $12,5 \mathrm{~mm}$ \\
\hline Standard FOV (H X V) & $62 \times 47$ \\
\hline Strobe & IR \\
\hline Connection Type & Cat5e/RJ45 \\
\hline
\end{tabular}

The main focus of these diagrams is their $3 \mathrm{D}$ representation of the trajectory. They are obtained using six cameras interfaced with a personal computer. The points shown in diagrams have been averaged and shown in Tab. 2. Based on these results, parameters of the proposed mechanism are constructed and its trajectory is shown in Fig. 13. In this figure, the trajectory of point E from the mechanism shown in 6 can be seen.

Table 2 Averaged data points coordinates

\begin{tabular}{|c|c|c|c|}
\hline No. & $X / \mathrm{mm}$ & $Y / \mathrm{mm}$ & $Z / \mathrm{mm}$ \\
\hline 1 & 237,115 & 1,98 & 1048,34 \\
\hline 2 & 222,206 & 94,13 & 1062,19 \\
\hline 3 & 217,433 & 181,96 & 1068,91 \\
\hline 4 & 217,337 & 273,17 & 1067,04 \\
\hline 5 & 223,901 & 371,453 & 1052,86 \\
\hline 6 & 234,117 & 480,131 & 1040,33 \\
\hline 7 & 244,473 & 597,334 & 1046,58 \\
\hline 8 & 262,889 & 694,536 & 1055,8 \\
\hline 9 & 273,042 & 784,479 & 1063,69 \\
\hline 10 & 276,972 & 875,98 & 1058,02 \\
\hline 11 & 273,729 & 976,964 & 1039,8 \\
\hline 12 & 263,484 & 1085,663 & 1031,69 \\
\hline 13 & 251,024 & 1200,468 & 1048,17 \\
\hline 14 & 233,915 & 1297,064 & 1059,56 \\
\hline 15 & 224,114 & 1382,501 & 1063,27 \\
\hline 16 & 219,519 & 1471,941 & 1058,73 \\
\hline 17 & 221,783 & 1571,115 & 1043,9 \\
\hline 18 & 228,034 & 1686,44 & 1029 \\
\hline 19 & 233,477 & 1813,915 & 1034,83 \\
\hline 20 & 247,108 & 1917,379 & 1046,59 \\
\hline & & & \\
\hline
\end{tabular}

The results are compared for a 2,5 step length. Data from this experiment show that there is coincidence between COM coordinates obtained from a normal human and mechanism walk. It provides approximately equal transition of COM and it balances body weight. By passing from one foot to the other, body weight is shifting, thus preventing patient from flipping over.

The future scope of the experiments will involve velocities and accelerations to improve the mechanism, i.e. to gain a better transition of body weight. In this case, body's mass has equal transition from one side to the other, but the aim is to move mass from one side to the other at such a speed so as to avoid the effects of inertia forces.

\section{CONCLUSION}

In this paper, kinematics and experimental analysis of a single walker mechanism is carried out in order to characterize the mechanism performance. More specifically, this work presents the mechanism embedded in a mechanical walker by which the balancing of a human mass centre is accomplished. This mechanism, as an original solution, has been calculated, 3D modelled and its operation simulated, analysed and numerically and graphically described. Kinematics equations of the proposed walking mechanism are formulated for a situation when a body becomes a balance in a supporting standing and walking condition. This is solved in SolidWorks and gained results can be used for further kinematic optimization of the mechanism.

An experimental model of the proposed mechanism has been also developed, and the test for the situation of ground walking has been accomplished. Finally, the motion of the chosen referent point on the mechanical walker obtained by the simulation (Fig. 13) is compared with the motion of the corresponding referent point on the human body acquired by the Vicon Motion Capture system. This system is infrared marker-tracking equipment installed at the Faculty of Technical Sciences in Novi Sad and offers millimetre resolution of 3D spatial displacements. The results of this comparison revealed that trajectories of the chosen referent points on the mechanical walker and human body are almost overlapped. Thus, it has been proven that the mechanism proposed in this paper is capable to balance the mass centre of a human body correctly.

In the end, we emphasize the importance, advantages and novelty of the proposed solutions. First of all, the exposed mechanism is suitable for human rehabilitation purposes. For instance, it can be applied for medical rehabilitation of paraplegic persons whose spinal cord is injured. Secondly, the walker device with the balanced mass centre can be supplied with the feet of a smaller size. And finally, but not of least importance, the walker described in this paper generates more pleasant walking movement, similar to the physiological bipedal motion. The novelty of the design structure, also important to accentuate, is that this mechanical walker is supplied only by one motor which drives both embedded mechanisms and consequently, there is no need for a complex command and control algorithm. 


\section{REFERENCES}

[1] Hirukawa, H., Hattori, S., Kajita, S., Harada, K., Kaneko, K., Kanehiro, F.,... \& Nakaoka, S. (2007, April). A pattern generator of humanoid robots walking on a rough terrain. InRobotics and Automation. 2007 IEEE International Conference on (2181-2187). IEEE. https://doi.org/10.1109/ROBOT.2007.363644

[2] Khatib, O., Warren, J., De Sapio, V. \& Sentis, L. (2004). Humanlike motion from physiologically-based potential energies. On Advances in Robot Kinematics, Springer Netherlands, $145-154$ https://doi.org/10.1007/978-1-4020-2249-4_16

[3] Mansard, N., Stasse, O., Chaumette, F. \& Yokoi, K. (2007). Visually-guided grasping while walking on a humanoid robot. IEEE International Conference on Robotics and Automation, 3041-3047. https://doi.org/10.1109/ROBOT.2007.363934

[4] Khatib, O. \& Sentis, L. (2005). Synthesis of whole-body behaviors through hierarchical control of behavioral primitives. International Journal of Humanoid Robotics, 2(4), 505-518. https://doi.org/10.1142/S0219843605000594

[5] Monková, K., Monka, P., Hloch, S. \& Valíček, J. (2011). Kinematic analysis of quick-return mechanism in three various approaches. Tehnicki vjesnik-Technical Gazette. 18(2), 295-299.

[6] Ivandic, Z., Ergic, T. \& Kljajin, M. (2009). Welding robots kinematic structures evaluation of based on conceptual models using the potential method. Tehnicki vjesnikTechnical Gazette, 16(4), 35-45.

[7] Collins, S. H., Wisse, M., \& Ruina, A. (2001). A threedimensional passive-dynamic walking robot with two legs and knees. The International Journal of Robotics Research, 20(7), 607-615. https://doi.org/10.1177/02783640122067561

[8] Koceska, N., Koceski, S., Beomonte, P., \& Durante, F. (2011). Gait Training using Pneumatically Actuated Robot System, InTech, 224-238.

[9] Andrejević, R., Šiniković, G., Stojićević, M., Stoimenov, M., Miladinović, L., Popkonstantinović, B.,.. \& Stankovski, S. (2013). A novel walker with mechanically established walking and standing mechanism. Tehnički vjesnik-Technical Gazette, 20(6), 927-931.

[10] Vukobratović, M., \& Borovac, B. (2004). Zero-moment point - thirty five years of its life. International Journal of Humanoid Robotics, 1(01), 157-173. https://doi.org/10.1142/S0219843604000083

[11] Stojićević, M., Stoimenov, M., Petrović, D. \& Bugarić, U. (2015). Mechanism with approximately straight-line path of dyad. The $14^{\text {th }}$ IFToMM World Congress, Taipei, Taiwan, October 25-30, 2015 Oral Session 86-3. https://doi.org/ 10.6567//FToMM.14TH.WC.0S2.028

[12] Remez, E. (1935). A brief explanation of the reasoning of Chebyshev concerning the approximation of functions. Kiev : Izd. Akad. Nauk UkSSR.

[13] Artobolevskii, I. (1945). Russian mechanism science. Moscow : Vestnik Akad. Nauk. SSSR.

[14] Rašković, D. (1964). Osnovi teorije mehainzama. Belgrade : Zavod za izdavanje udžbenika.

[15] Hsu, M. H., Chen, Y. G., Liu, J. Y. \& Hsieh, L. C. (2007). On the Study of Ellipsograph Mechanisms. The $12^{\text {th }}$ World Congress in Mechanism and Machine Science IFToMM2007, June 17-21, Besançon, France, Paper No. A925.

\section{Contact information:}

Miša STOJIĆEVIĆ, M.Sc.

Mechanical Engineering Faculty,

University of Belgrade,

Kraljice Marije 16, 11000 Beograd, Serbia

E-mail: mstojicevic@mas.bg.ac.rs

\section{Miodrag STOIMENOV, Ph.D.}

Mechanical Engineering Faculty,

University of Belgrade,

Kraljice Marije 16, 11000 Beograd, Serbia

E-mail: mstoimenov@mas.bg.ac.rs

\section{Zorana JELI, Ph.D.}

Mechanical Engineering Faculty,

University of Belgrade

Kraljice Marije 16, 11000 Beograd, Serbia

E-mail: zjeli@mas.bg.ac.rs 